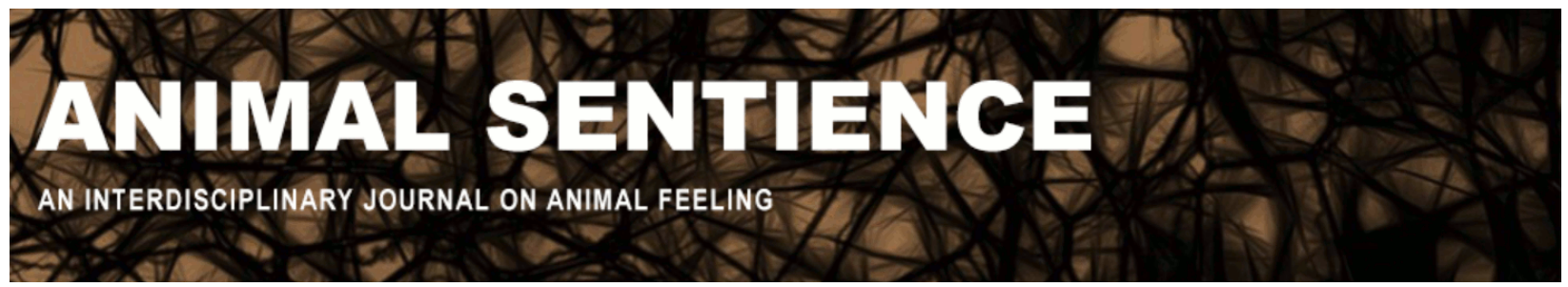

Pepperberg, Irene M. (2019) Animal sentience is not enough to motivate conservation. Animal Sentience 23(22)

DOI: $10.51291 / 2377-7478.1396$

Date of submission: 2019-02-03

Date of acceptance: 2019-02-18 (c) 


\title{
Animal sentience is not enough to motivate conservation
}

Commentary on Chapman \& Huffman on Human Difference

\author{
Irene M. Pepperberg \\ Research Associate in Psychology \\ Harvard University
}

\begin{abstract}
Chapman \& Huffman suggest that humans' views of their own superiority are a source of their callousness toward the environment. I do not disagree but point to a number of other issues that must be addressed for conservation efforts to succeed.
\end{abstract}

Keywords: animal sentience, animal conservation, nonhuman sentience

Irene M. Pepperberg is currently a Research Associate (Psychology) at Harvard, and has authored over 150 peer-reviewed journal articles and book chapters, and the NY Times bestseller Alex \& Me. For over forty years, she has trained grey parrots to use English speech referentially and employed this communication code to examine their intelligence. Website

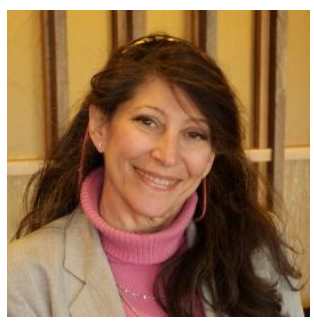

Chapman \& Huffman (2018) (C \& H) make a well-reasoned plea for the rights of nonhumans. They describe how humans are destroying the environment through their food and energy choices, show how nonhumans' intellect often matches or occasionally exceeds that of humans, and tie the two discussions together by arguing that lack of human superiority means humans should not have exclusive nor even primary rights to the use of natural resources. C \& $\mathrm{H}$ then use their conclusion to argue for needed changes. I find little in their stated material with which to take issue. My commentary (unlike most others) will thus focus not on human/nonhuman similarities or differences, but on the issues the authors raise with respect to conservation.

I do not disagree with C \& H's main points: I admit that I have also used the argument that humans are more likely to protect species with which they can find common attributes, such as intelligence. There is also no question that the more attention average (mostly first-world) citizens pay to the origins of the foods they eat, the ingredients in their processed foods, and to even small changes in their energy expenditures (e.g., using LED bulbs, unplugging unconnected chargers) that can make significant differences - particularly when multiplied by the actions of millions of people - the healthier will be our planet. However, conserving our environment requires additional actions beyond increasing human respect for nonhumans and consequent decisions to choose a more plant-based diet or more energy-efficient means of living. Conservation problems are multifaceted and require far more complex solutions. I would have liked to hear more from $\mathrm{C} \& \mathrm{H}$, with respect to 'boots on the ground', life-as-it-is-reality-check issues in addition to the stark numbers presented.

I thus raise the following issues: Many of those eating bush meat or trapping for the pet 
trade have no choice - either they hunt and trap or their families starve (see Ripple et al., 2016). People kill each other for less pressing reasons; even a belief in nonhuman intelligence/sentience won't trump personal survival. Many countries have few or no funds to support forest rangers (not only their salaries, but also appropriate tools and equipment) to enforce whatever environmental protections already exist; in many cases, funds earmarked for such activities are lost to graft and corruption (note World Wildlife Fund, 2015). Setting aside land for preservation may encroach on the livelihoods of the poorest (note Lee \& Priston, 2005).

And how much land must be set aside? We now know enough about edge-effects to realize that in many instances, small patches may either be useless for some species or favor certain species over others and thus cannot fully save the native ecosystem (see discussion in Harris, 1988). For some third-world citizens, nonhumans are direct competitors for their subsistence diets, whether in terms of foraging for the same foods or acting as a source of predation on their herds (Lee \& Priston, 2005; Naughton-Treves, 1998); again, understanding nonhuman intelligence will not alter humans' views on the need for their own survival.

Eco-tourism may make up for some of the loss of income engendered by, for example, the banning of safari hunting and poaching, but eco-tourism also stresses the environment (e.g., overuse and degradation of habitat, increase in costs of local goods, impact on climate with respect to travel to these destinations); however, eliminating eco-tourism would eliminate what is often an important source of revenue (see discussion in Saarinen, 2006).

Occasionally, conservation success engenders substantive negative issues, as in wildlife encroaching into urban areas to the extent that once-threatened species become pests that are targeted for destruction rather than conservation; here nonhuman intelligence may become a negative rather than positive attribute in terms of nonhumans' ability to defeat barriers that humans erect for their own protection (see review in Mormile \& Hill, 2017). I also note that even though nonhuman intelligence is often used as a 'selling point' by entities whose only goals are purportedly to serve conservation efforts, intergroup competition (e.g., in fundraising efforts or attribution of credit) may make achieving the goals of any one of these entities more difficult.

Specifically, targeted education about the value of various endangered species and habitats is necessary but far from sufficient to promote conservation. Citizens of all economic strata must be involved. For many indigenous people to care about conservation, their basic needs (food, shelter, health) must first be addressed, and in ways that do not require additional destruction of the environment. Even what appear to be simple human actions for better-quality lives may have serious downstream effects: For example, let us assume that mosquitos fail to demonstrate any level of cognitive processing, so the elimination of malaria-bearing insects would not only improve the immediate health of millions of humans, but it would also be guilt-free. However, what of the nonhumans for whom mosquitoes constitute a majority of their diet, and the rest of the dependent food chain?

Adherence to C \& H's suggestions would probably improve the overall well-being of our planet, but the steps they put forth are only a partial solution to a complex conundrum. 


\section{References}

Chapman, C.A., \& Huffman, M.A. (2018). Why do we want to think humans are different? Animal Sentience 21(3).

Harris, L.D. (1988). Edge effects and conservation of biodiversity. Conservation Biology, 2, 330332.

Lee, P.C., \& Priston, N.E.C. (2005). Human attributes to primates: Perception of pests, conflict and consequences for primate conservation. In J.D. Paterson \& J. Wallis (Eds.), Commensalism and conflict: The human-primate interface (pp. 1-23). Norman, OK: American Society of Primatology.

Mormile, J.E., \& Hill, C.M. (2017). Living with urban baboons: Exploring attitudes and their implications for local baboon conservation and management in Knysna, South Africa. Human Dimensions of Wildlife, 22(2), 99-109.

Nasi, R., Brown, D., Wilkie, D., Bennett, E., Tutin, C., van Tol, G., \& Christophersen, T. (2008). Conservation and use of wildlife-based resources: The bushmeat crisis. Secretariat of the Convention on Biological Diversity and Center for International Forestry Research and Center for International Forestry Research.

Naughton-Treves, L. (1998). Predicting patterns of crop damage by wildlife around Kibale National Park, Uganda. Conservation Biology, 12(1), 156-168.

Ripple, W.J. et al. (2016). Bushmeat hunting and extinction risk to the world's mammals. Royal Society Open Science, 3, 160498.

Saarinen, J. (2006). Traditions of sustainability in tourism studies. Annals of Tourism Research, 33(4), 1121-1140.

World Wildlife Fund. (2015). Working together to combat illegal and unsustainable wildlife trade corruption in wildlife conservation: A primer. 